\title{
Imaging Mueller matrix ellipsometry setup for optical nanoform metrology
}

\author{
Tim Käseberg ${ }^{1,}$, Jana Grundmann ${ }^{1}$, Johannes Dickmann ${ }^{1,2}$, Stefanie Kroker ${ }^{1,2}$, and Bernd Bodermann ${ }^{1}$ \\ ${ }^{1}$ Physikalisch-Technische Bundesanstalt, Bundesallee 100, 38116 Braunschweig, Germany \\ ${ }^{2}$ Laboratory for Emerging Nanometrology, Technische Universität Braunschweig, Langer Kamp 6a/b, 38106 Braunschweig, Germany
}

\begin{abstract}
We designed, realized, and characterised an imaging Mueller matrix ellipsometry setup for the pixelwise measurement of the Mueller matrices in microscope images. Our setup is capable of performing measurements in reflection as well as in transmission in a broad range of angles of incidence for wavelengths between $400 \mathrm{~nm}$ and $700 \mathrm{~nm}$. We compared measurements of specially designed nanostructured samples with AFM and SEM measurements as well as with numerical simulations using the finite element method.
\end{abstract}

\section{Introduction}

The reliable production of structures at the nanometre scale remains a crucial issue in modern nanotechnology and it relies on the precise characterisation of the structures using fast and non-invasive measurement techniques. One popular technique for this task is Mueller matrix ellipsometry, which is a non-imaging optical technique that allows for the determination of the polarizing characteristics and by this to derive information about structural features of a sample [1,2]. The polarization state of light can be fully described using Stokes vectors. The $4 \times 4$ elements of the Mueller matrix provide the mathematical transform for these Stokes vectors of incident and outgoing light. As an integral measurement method, ellipsometry is capable of examining a rather large integration area, while restricting its measurement results to mean values of the whole illumination area only. Given usually large beam sizes in common ellipsometric setups, these areas rarely fall below several $10 \mu \mathrm{m}$ in diameter, whereas applications such as in semiconductor industries deal with much smaller measurement targets.

This limitation can be overcome by upgrading the classic ellipsometric setup with an imaging system. By this, the Mueller matrix of the sample can be determined for each pixel in an image, allowing for evaluations of sample features within much smaller interaction areas than the illumination area. This can be complemented by the investigation of off-diagonal elements of the Mueller matrix which have been shown to provide additional information about subwavelength sized features [3].

Our investigations include the construction of such an imaging ellipsometry setup and its application for the characterisation of different metastructure samples $[4,5]$. Our setup provides a flexible composition that allows for

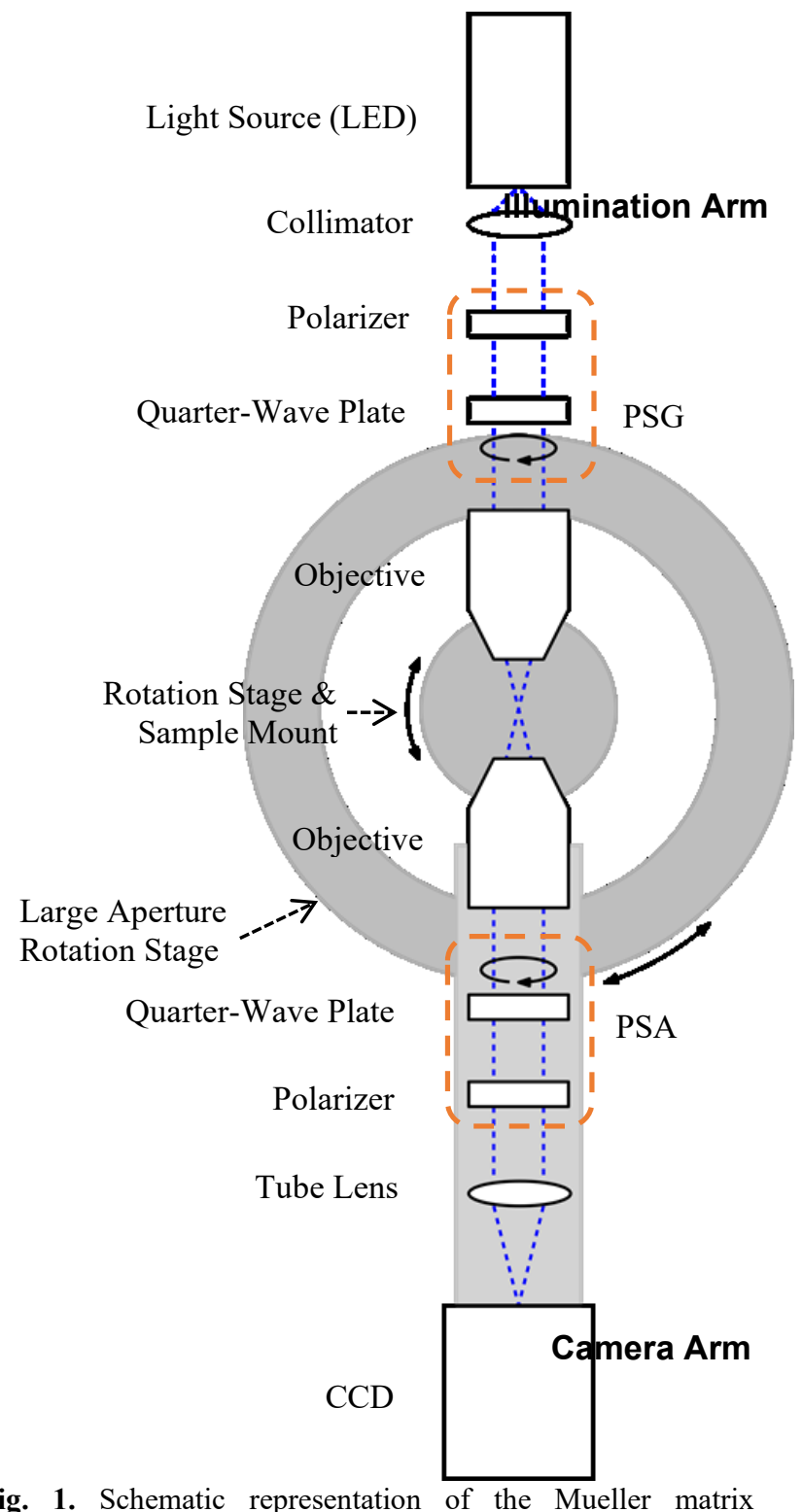

Fig. 1. Schematic representation of the Mueller matrix microscope setup.

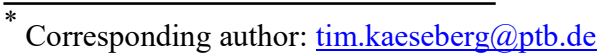


measurements in both reflection and transmission for a wide range of angles.

\section{Experimental Setup}

The setup of our Mueller matrix microscope is sketched out in figure 1. It is composed of two separate arms: a fixed illumination arm and a rotating camera arm. The illumination arm consists of a white light LED, limited to single wavelengths using bandpass filters, as well as a collimating lens and a polarisation state generator (PSG) system. The PSG contains a linear Glan-Thompson polarizer and a rotating quarter-wave plate. An objective focuses the light onto the sample which is mounted on top of a couple of linear and rotation stages for the precise adjustment of the sample in all degrees of freedom. The camera arm is attached to a rotation stage with a large aperture of $30 \mathrm{~cm}$ diameter with the same axis of rotation as the smaller sample rotation stage at its centre. An infinity corrected objective with a long working distance of $25.5 \mathrm{~mm}$, a magnification of $50 \mathrm{x}$ and a numerical aperture of $\mathrm{NA}=0.42$ combined with a tube lens creates an image of the sample that is collected by a cooled monochromatic CCD camera at the end of the camera arm. In between the objective and the CCD, a polarization state analyser (PSA) identical to the PSG but in reverse order is positioned. The setup forms a conventional dualrotating retarder Mueller matrix ellipsometer where the two retarders are rotated at different angular frequencies at a certain ratio. By this, the whole Mueller matrix can be determined for each pixel in the obtained image. To avoid collisions of the arms, limit switches restrict their movement. The setup as well as the measurement procedures are controlled by a LabVIEW interface.

\section{First Measurements}

The mechanical and optical components of the measurement system have been characterized. The system can be used to investigate in the polarizing properties of arbitrary samples. Figure 2 shows the very first preliminary Mueller matrix image recorded as a test of our setup for $11 \mu \mathrm{m}$ wide lines in transmission mode at normal incidence. As a first step, we examine plasmonic lenses that can be used as resolution enhancement devices. Our goal is to include the use of these structures in advanced Mueller matrix ellipsometry setups for an enhanced sensitivity to structures at the nanometre scale. Therefore, we produced a set of plasmonic lenses for applications at several visible wavelengths using electron beam lithography methods. First tests with these lenses were performed at our setup. Additionally, we developed concepts on the combination of ellipsometric setups and plasmonic lenses. To further examine the connection between subwavelength sized structural features and offdiagonal Mueller matrix elements, we designed and fabricated a set of geometrical samples with different shapes, ranging between squares and circles [4]. The measurements of all these distinct structures are compared to numerical simulations, performed with the finite element tool JCMsuite [6]. Apart from these structures, measurements of samples with well-known properties at our setup were compared to measurements with scanning electron microscopy (SEM) and atomic force microscopy (AFM) setups.

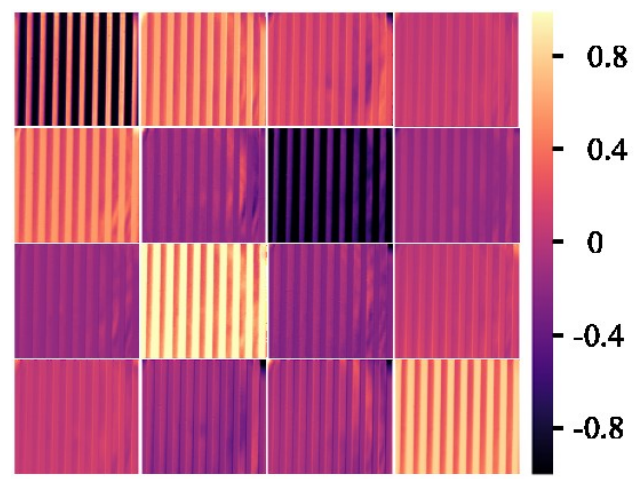

Fig. 2. First preliminary Mueller matrix image of $11 \mu \mathrm{m}$ wide lines. Each image contains the pixelwise values of one element of the $4 \times 4$ Mueller matrix.

This work is funded through the project 17FUN01 "BeCOMe" within the Programme EMPIR. The EMPIR initiative is cofounded by the European Union's Horizon 2020 research and innovation program and the EMPIR Participating Countries

\section{References}

1. M. Losurdo, K. Hingerl, Ellipsometry at the Nanoscale (Springer, Berlin, 2013)

2. A. Diebold, A. Antonelli, N. Keller, APL Materials 6, 058201 (2018)

3. N. Kerwien, On the influence of polarization effects in microscopic image formation, Berichte aus dem Insitut für Technische Optik 58 (2007)

4. T. Käseberg, J. Dickmann, T. Siefke, M. Wurm, S. Kroker, B. Bodermann, Proc. SPIE 11057, 110570R (2019)

5. T. Käseberg, T. Siefke, S. Kroker, B. Bodermann, Meas. Sci. Technol. (manuscript accepted), https://doi.org/10.1088/1361-6501/ab7e6b

6. JCMsuite, JCMwave GmbH, Berlin. https://www.jcmwave.com/ 